\title{
A Multi Robotic Assistant System (MRAS): A develop- ment approach with application to the ageing society
}

\author{
Thomas Linner *, Christos Georgoulas, and Thomas Bock \\ Department of Architecture, Building Realization and Robotics Lab, Technical University \\ Munich, Germany \\ * Corresponding author (thomas.linner@br2.ar.tum.de)
}

\begin{abstract}
Purpose Our purpose was to develop, by combining architectural and robotic elements, a system that assists elderly people to live self-sufficiently at home. Unlike many other assistance systems our system exploits the ceiling space in order to avoid collisions with conventional living functions. Method Elderly people face numerous limitations in their everyday life. The simplest regular tasks a healthy individual performs appear quite different from the perspective of the ageing adult. Healthcare and mobility usually appear as distant services in cases of isolated individuals or lack of transportation due to disability. This paper deals with the development of a novel multi-robot assistant system (MARS), which can be used to provide indoor quality services to the ageing society. In order to realize the MARS concept the following development approach was used: (i) research and identification of needs; (ii) definition of requirements; (iii) identification of technologies and processes; (iv) initial concept; (v) experiments in real environment; and (vi) final concept and further development roadmap. Results \& Discussion A ceiling suspended robotic system is proposed, navigating with the aid of a grid-type rail system among various rooms within a residence. This enables efficient space utilization since it occupies the redundant area of the ceiling. Various types of services can be provided by the proposed system such as health monitoring, infotainment, communications, problem solving, movement assistance, etc. A vision system aids in obtaining accurate information on the location of the individual within the residence, and in guiding the whole system during the operation of the required tasks. A series of experiments were conducted in order to define the proposed system specifications. Verification experiments in a real residence with three different individuals were performed, in order to calculate the forces applied to the human body while sitting and standing up (Figure 1). This type of movement is the most awkward and laborious for elderly people. MRAS can assist elderly people by using a flexible pulling wire which can be easily either grabbed by the user or fastened onto.
\end{abstract}

Keywords: ambient integrated robotics, architecture, multimorbidity, stereo vision

\section{INTRODUCTION}

Demographic change studies, dealing with elderly population growth, reveal a relatively rapid increase, and it is expected that in the next half century the proportion of elderly people will increase from $7 \%$ in 2000 to $16 \%$ in $2050^{3}$. The majority of the elderly people face health problems that oblige them to remain at home and to depend on another person. Hence, it is essential to focus on the elderly people needs and work on robotic devices that will provide them an independent and dignified everyday living. Research community has focused towards this direction and has been presented assistive devices that aim to facilitate the daily activities of elderly people, such as for example: PPMA system for personal mobility aid and health monitoring ${ }^{4}$, robotic walker for navigation and obstacle avoidance ${ }^{5}$, MAT robot for domestic activities (housekeeping, eating, grooming, entertainment, etc. $)^{6}$.

Several ceiling suspended lift systems for elderly and disabled people are currently on the market ${ }^{7}$. All of these systems impose several limitations, since they are restricted to specific path ways, are expensive and require major modification within a flat. Addition- ally, they can only be used for one purpose: the purpose of lifting persons. Furthermore, several researchers are working on mobile and dexterous service robots that operate on the ground ${ }^{8}$. Those systems often can assist with multiple tasks but their area of operation is restricted as they are bound to the ground and complex interactions with human beings and other obstacles as furniture are unavoidable. The ceiling can be identified a perfect space within any environment for the guided and efficient operation of a service robot. The concept of a ceiling robot within an environment has already been explored by Sato ${ }^{9}$.

In this paper a combination of existing approaches is proposed, leading to a novel multi robot assistant system (MRAS). The system aim is to assist in Activities of Daily Living (ADLs) ${ }^{10}$, and is especially oriented to the needs of the aging society. The system has the ability to move in all rooms of the residence without occupying floor space, since it moves on the ceiling using a rail type grid system. The system is multifunctional accomplishing various activities (e.g. health monitoring, delivering prescribed medication at appropriate times and dosages, info- 
tainment, communications, etc.). Moreover, the system provides the ability to aid the elderly people in functional transfers, i.e. move from a sitting position to a standing position.

\section{MRAS CONCEPT AND ITS MAIN FEATURES}

MRAS concept presents a multi robot assistant system based on the ergonomics concept, since it utilizes the ceiling area which is unexploited. Thus, it does not occupy area of the floor, allowing elderly people to easily move within a residence, especially when considering people using mobility assistive devices such as wheelchair and rollators. Additionally, it can be customized, in terms of functionality, in order to adapt to the exact needs of each user, since the weaknesses and health problems of each user differ.

One common problem that elderly people have to face is the accidents in the residence especially due to falls. In order to aid elderly people with eye disorders, the robot system is able to automatically adjust the lightning conditions within a residence, depending on the time of day and the degree of darkness in the room.

The MRAS also incorporates an air purifier that reduces the air contaminants, improving the quality of living of people with respiratory problems. In addition, this system in combination with the lightning control system can provide the adequate environment for sleep, since the majority of the elderly society faces sleep disorders.

Moreover, the MRAS integrates a medication dispenser. The system dispenses medication to the individuals at corresponding time intervals and in the appropriate dosage. In this way, the individuals receive medication in a properly manner, avoiding mistakes and without missing out doses. Moreover, a measuring system monitors the individual's vital signs, through measuring blood pressure and blood glucose levels at daily intervals. The obtained data are compared to the allowed limit values. In case that the measured values overcome the allowed limit values, an emergency signal is transmitted to a registered physician. The weekly values are also transmitted to a physician in order to be informed about the individual's weekly health status.

MRAS can also assist elderly people in their functional transfers, by using a flexible pulling wire which can be easily either grabbed by the individual or fastened onto them using a wearable harness. More specifically, the system can move the elderly people from a sitting position to a standing position or from a lying position to a sitting or standing one. The value and the direction of the force that must be applied to pull the individual by the wire in order to change his/her position is achieved by the calculation of the external force in each position step as shown in Figure 1. Experiments with three different individuals which move from sitting position to standing position were performed, in order to estimate the required forces applied, and define the necessary payload of the system. The movement has been separated in 5 "sub-movements" as shown in Figure 1.

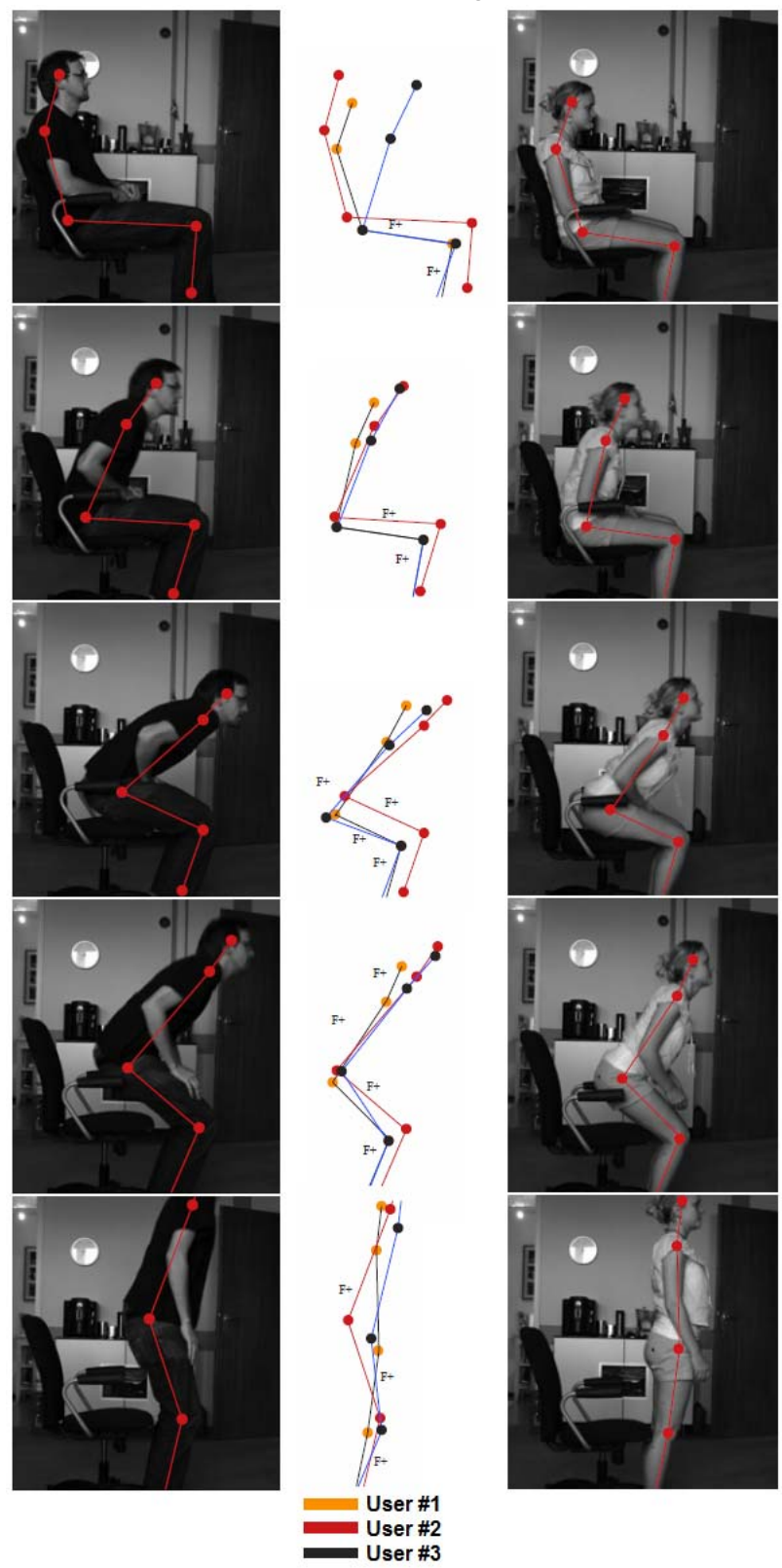

Fig.1. Experiment for calculating the amount of forces individuals are subjected to, during functional transfers

\section{Mechanical Concept}

The mechanical concept of the proposed MRAS was defined after considering the following terms:

- Technology,

- Modularity,

- In-Exchangeability,

- Low Maintenance.

Next Generation robotics could help ease the partially intricate life of the elderly. The addition of features and availability would result in a reduced need for 
caretakers, and raise the independence of elderly people, in order to be able to perform easily the ADLs and remain more at their homes. With a wide spectrum of exchangeable robotic elements, such as manipulators and actuators, a more individual robotic system is feasible. Each person has different needs, and thus a robotic system could be adjusted to fit explicit needs.

By following a modular structure, the robotic system would not only be more cost efficient but also give the possibility of enhanced service delivery. These features could be updated and exchanged upon request, offering the possibility of an increased diversity through third party development. The use of a modular system also introduces an adaptive concept and lessens the chance of cost ineffective and complex component exchanges.

Equipped with Plug-n-Play functionality, the proposed MRAS would be able to have almost infinite feature availability through third party developers. Ease of component exchange and installation, also reduces installation errors. The idea is to keep the robotic system as flexible as possible without loss of performance.

A system needs to require low maintenance in order to be considered efficient. This not only reduces expenses but also overcomes possible hassle between the customer using the system and the manufacturer companies that cover its warranty. A low maintenance health care system can be in the future provided to the elderly by the healthcare provider sector, if the required procedure involves only mounting, i.e. a plug-n-play behavior.

Fig.2. Proposed System Overview

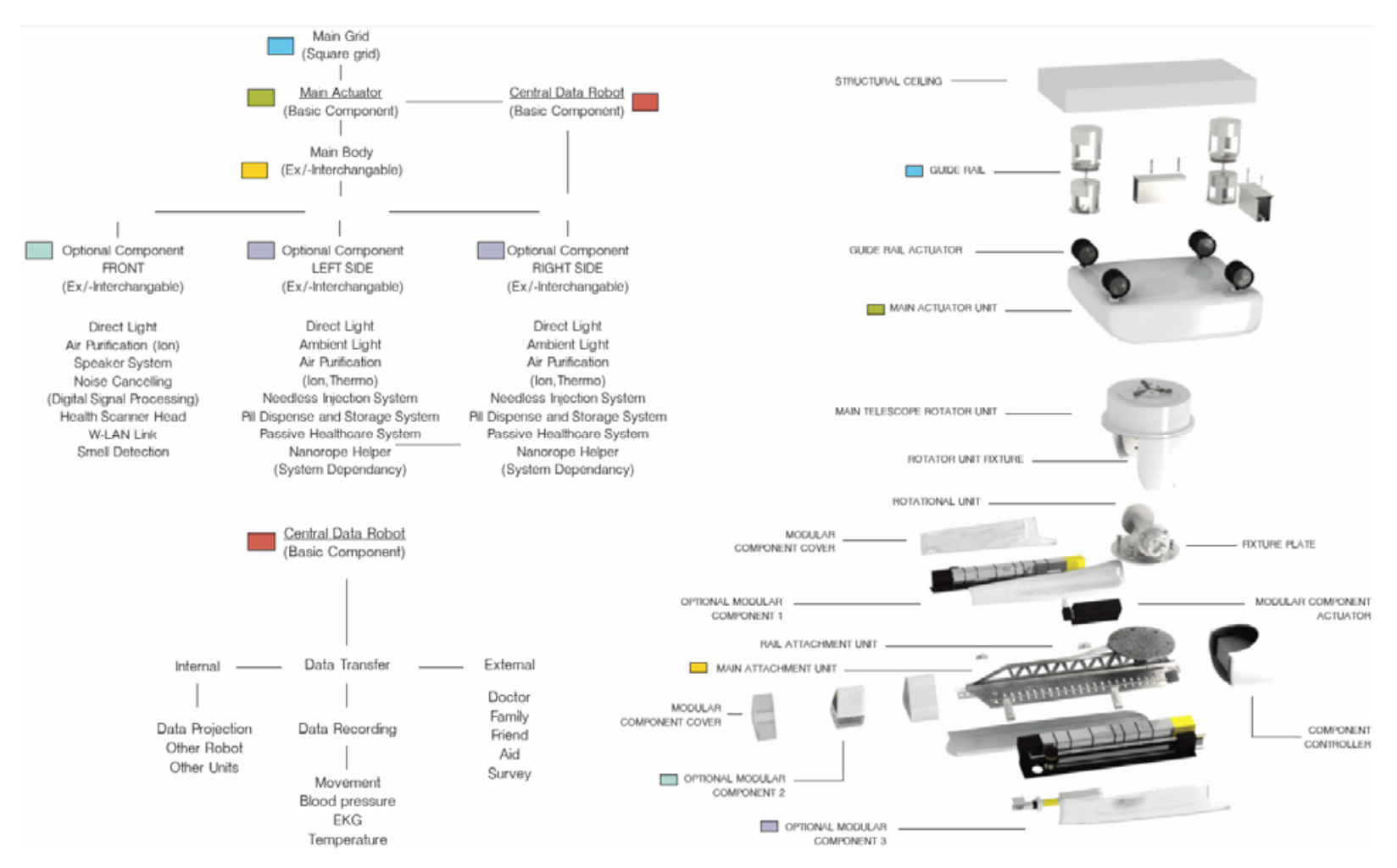

\section{DESIGN APPROACH}

The MRAS design followed the aforementioned considerations in order to comply with the required specifications, and enable a system that will efficiently address the ageing society needs. A system overview is provided in Figure 2.

The proposed system is divided into 3 main components:

- The Main Grid (Rectangular Arrangement Grid)

- $\quad$ The Main Actuator (Basic Component)

- $\quad$ The Main Body (Ex/-Interchangeable)

\section{Main Grid}

The main grid comprises a rail type grid infrastructure attached to the ceiling, which provides the means for mobility within the various areas of the residence. According to the final dimensions of all system modules, an appropriate grid structure is attached to the ceiling, consisting of rectilinear steel rails, arranged in a rectangular mesh, in order to allow the robotic system to be able to move nearly to all possible locations within each operating area.

\section{Main Actuator}

The main actuator comprises the mobility element of the overall system. It is responsible for directing the structure to the correct location, utilizing the steel rail grid attached to the ceiling. The main actuator is the central core of the MRAS. The actuator itself uses spherical rubber and steel wheels to navigate the grid. The form and buildup of the wheels allows a directional navigation in both $Y$ and $X$ direction. The software algorithm used to navigate the grid could be 
based on the Cartesian system. The information exchange to and from the main actuator is transmitted by wireless LAN. A charging station located at a predefined position of the grid, allows the internal $\mathrm{Li}$ ION battery of the main actuator to be charged, while it is docked at the charging point. The internal battery is used as a backup alternative power supply mode, in cases of power failures and blackouts. While operating in normal power mode, the main actuator is supplied by the rail grid structure, which is interfaced to the main power supply of the apartment.

In order for the main actuator to be able to progress through the rectangular arranged grid, a sort of guiding mechanism needs to be implemented. At all intersection points of the rectilinear rails, a ball bearing arrangement was designed in order to successfully guide the main actuator module into the required directions, Figure 3. Once the main actuator wheels are positioned within the intersection points, the ball bearing arrangements rotate them by 90 degrees in order to align them with the perpendicular rail. Thus, the redirection of the main actuator to a perpendicular rail is achieved.

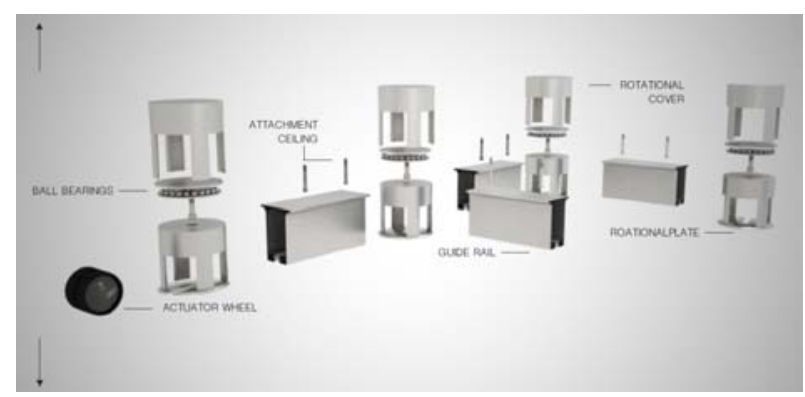

Fig.3. Rail guiding system

\section{Main Body}

The main body component comprises the endeffector module of the overall robotic element. It serves as the service delivery module, since it provides all the functionality and service delivery to the user. It is attached to the main actuator component via a telescope rotator unit, enabling rotation to all possible directions, allowing a 2-degrees-of-freedom operation.

The main body component follows a modular and customizable design, in terms of functionality and service delivery. The proposed concept focuses on enabling a fully modular and customized robotic system, in order to comply with low maintenance requirements, such as easy to upgrade and repair in case of malfunction. The design of the main body is depicted in Figure 4. A miniPC located under the rear cover, serves as the processing unit, handling all required processes and operations, as well as deploying the modular units of the proposed component.

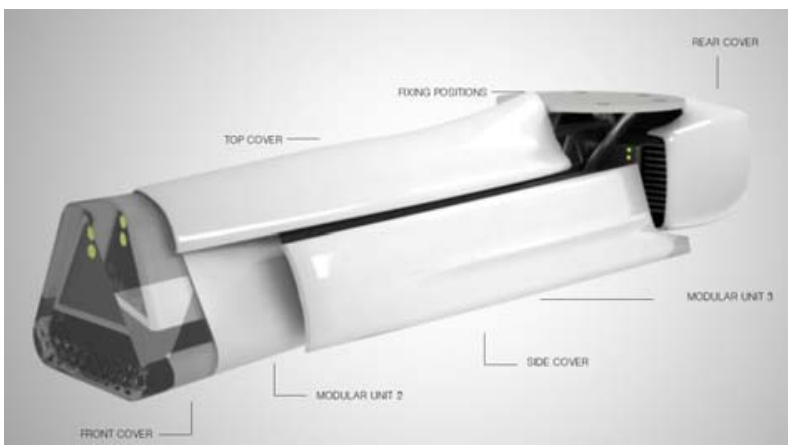

Fig.4. Main Body Component

\section{Proposed Functionality}

The functionality and features of the proposed MRAS focus on providing services assisting elderly people in daily activities within the home environment. Additionally, since the MRAS concept aims to increase the independence of elderly people and allow them to spend more time in their home environments, a series of applications and services contributing towards this goal, are proposed.

\section{Direct Light}

Direct lighting can be offered in areas of the home environment that low lightning conditions are detected, regulated by the main control module. Light regulation, can be performed not only according to brightness but also to color, depending on the time of day and the degree of darkness in the room. Deployable panels populated with an LED mesh can offer direct light according to the user requirement. Additionally the control unit can adjust the panel angle and direction to achieve optimum lighting conditions.

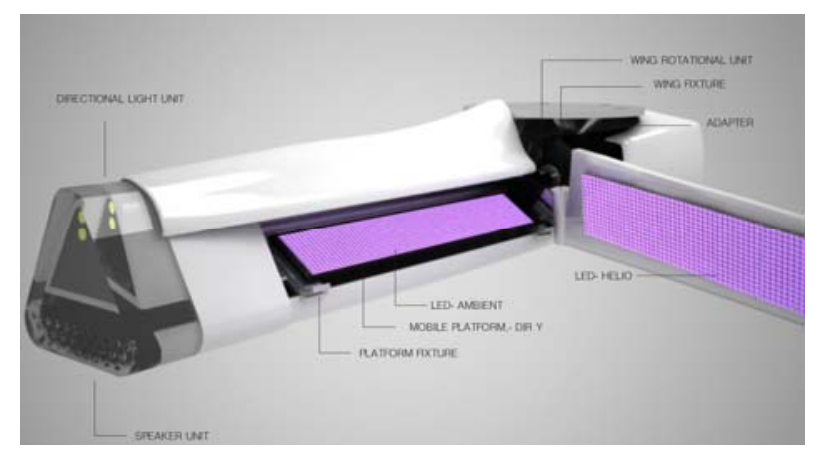

Fig.5. Direct Light Configuration

\section{Noise cancelling}

Through the use of ANC (Active noise control) and anti-phasing technology, repetitive noise patterns can be reduced or even "cancelled", leading to a more quite environment. The speaker unit attached to the main body module shown in Figure 5, can be used, controlled by a Digital Signal Processing (DSP) algorithm, to emit "Anti-Noise" signals to reduce or even cancel out city urban noises such as traffic, horns, alarm sirens, etc. The graphical depiction of Active Noise Reduction is shown in Figure 6. 


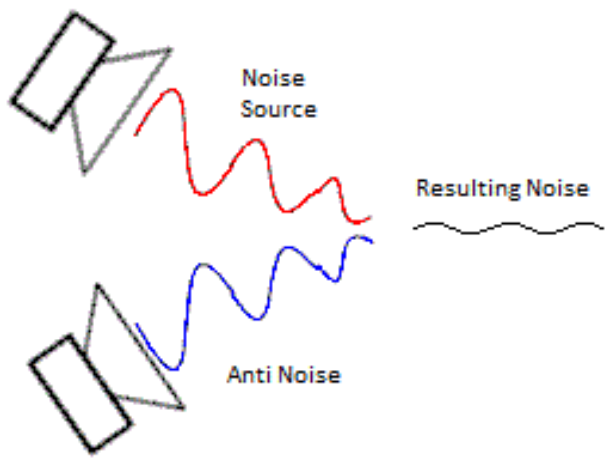

Active Noise Reduction

Fig.6. Active Noise Reduction (ACN) Principle

\section{Passive Healthcare}

The goal is the establishment of DIY (do it yourself) healthcare system. Depending on the technological progress, a microwave/laser emitter and receiver unit can be attached to the front part of the main robot. A passive measurement on the user blood pressure could be performed. The acquired measurement data can be exchanged via a digital communication network to a physician to allow tele-care and tele-consulting.

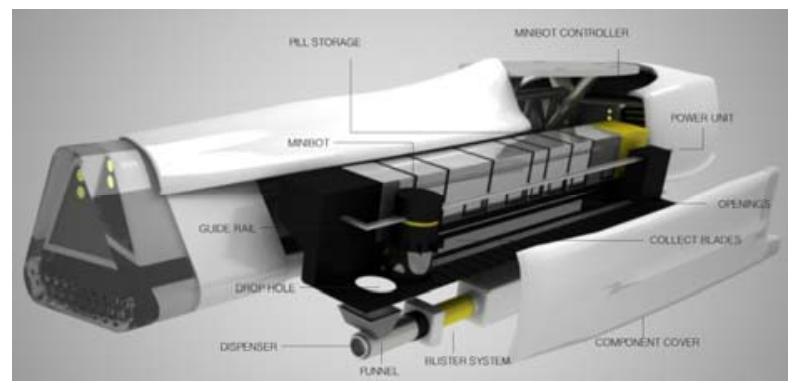

Fig.7. Passive Healthcare Configuration

Additionally a needless injection feature was concerned. Many elderly people require daily injections which are performed by designated health care personnel. A system can be attached as an add-on to MRAS in order to perform injections without requiring the presence of a specialized person. The technology preferred would be a solid micro-needle system that attaches to the skin and slowly injects the required medicine, which could be air-gunned from the main body component to the patient.

A medication dispenser mechanism was also considered as one of the offered services of the proposed concept. A medication cartridge unit, which can be filled at a pharmacy, facilitates the medication dispenser module. The cartridge can consist of several chambers, to allow various medications to be stored. A small robotic component travelling along a rail automatically collects the according medicine from the chambers and dispenses it through an opening into a small plastic package. Once the ap- propriate type and quantity of medication has been collected and places into the small plastic bag, the package is then dropped onto an area determined by the patient.

\section{Assistance in functional transfers}

The main body component can be configured to assist elderly people in their functional transfers (moving from a sitting position to a standing position or from a lying position to a sitting or standing one), by using a flexible pulling wire which can be easily either grabbed by the individual or fastened on them using a wearable harness. The proposed designed configuration can be seen in Figure 8.

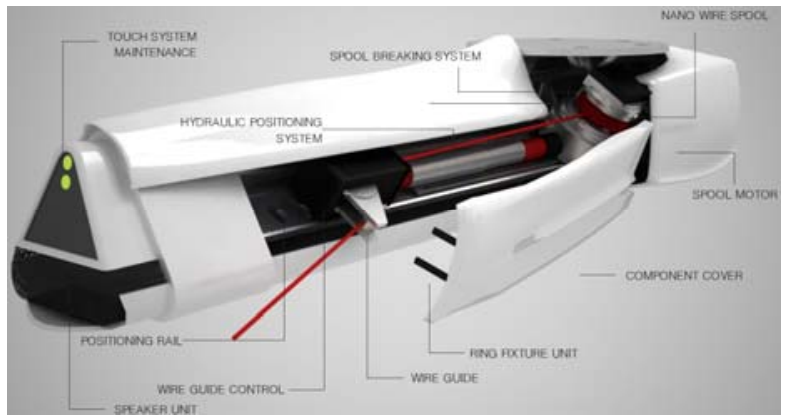

Fig.8. Functional Transfer Assistant

A wearable harness can be used in combination with the proposed configuration to assist individuals to perform functional transfers easier. A deployable wire can be lowered to the individual level, in order to be secured to the harness mechanism. Once secured firmly, a pulling mechanism retracting the wire can allow functional transfers to be performed in a more comfortable and supervised manner.

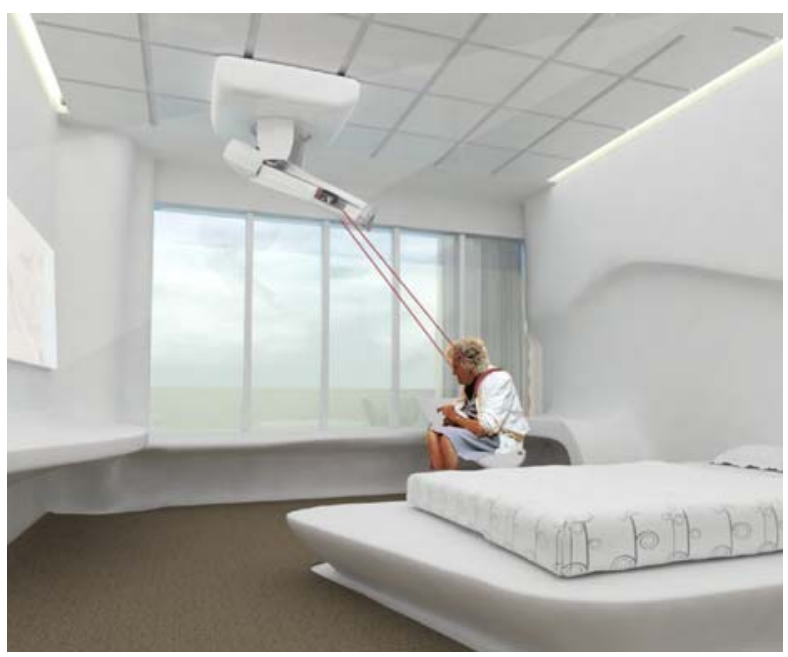

Fig.9. Functional Transfer Assistant

\section{HUMAN-MACHINE INTERACTION SCHEME}

The operation of the proposed MRAS concept and the service delivery can be controlled by a stereo vision camera system attached to the front of the main body component. The target person must be 
able to be detected by the robotic system in realtime, in order to allow efficient seamless and autonomous operation.

Depth perception is one of the important tasks of a computer vision system. Stereo correspondence by calculating the distance of various points in a scene relative to the position of a camera allows the performance of complex tasks, such as depth measurements and environment reconstruction ${ }^{11}$. The stereo correspondence problem comprises an active wide range of research. Many efforts have been made towards efficient solutions to address the various issues of stereo matching. As the improvements in computational resources steadily increase, the demand for real-time applications is getting compulsory $^{12}$. The most common approach for extracting depth information from intensity images is by means of a stereo camera setup. The point-by-point matching between the two images from the stereo setup derives the depth images, or the so called disparity maps $^{13}$. A stereo camera setup interfaced at the front of the main body component, can offer people detection, object recognition and many more.

A combined voice recognition module can increase the performance of the MRAS response capability. Vocal messages issued by the user, can be interpreted into commands to initiate services or to define required operating modes. Moreover, user queries regarding health status information, or lighting regulation, or even infotainment services can be used to control various functions and services. A humancomputer interaction scheme can thus be implemented.

\section{Conclusions}

A novel Multi Robotic Assistant System (MRAS) concept is proposed, focusing in assisting ageing society individuals within the home environment. The proposed system specifications were defined considering the need for a system realization that would comprise unattended health care services delivery, low maintenance costs, unobtrusive customization via modular add-ons provided by third party developers, autonomous operation, and human-machine communication features.

Verification experiments in a real residence comprising three different individuals were performed, in order to calculate the forces applied to the human body while performing functional transfers. These types of movements are the most uneasy and laborious for elderly people. MRAS can assist elderly people by using a flexible pulling wire which can be easily either grabbed by the individual or fastened onto. Elderly people usually utilize assistive devices such as wheelchairs and rollators. The requirement for enhanced space in order to freely move around their home environment is evident. MRAS concept efficiently addresses this issue, since it utilizes the un- occupied space of the ceiling. A rail type grid, to which the rest of the system is attached onto, is used in order to provide the necessary navigation paths within the operating area.

A stereo vision system along with a voice recognition algorithm is proposed to provide a human machine interaction scheme. Vision-based perception of moving people comprises one of the active research fields in computer vision. Monitoring people movements in complex environments, analyzing the resulting motion patterns and understanding people gestures corresponds to a high level of visual competence that can most appropriately be identified as Ambient Intelligence (Aml).

The proposed MRAS concept aims in providing a user-centric paradigm, supporting a variety of ageing society related daily living issues, operating pervasively, nonintrusive, and transparently.

\section{References}

1. Akker, M. van den, Buntinx, F., Metsemakers, J.F., Roos, S., Knottnerus, J.A., "Multimorbidity in general practice: prevalence, incidence, and determinants of co-occurring chronic and recurrent diseases", Journal of Clinical Epidemiology, Vol. 51(5), pp. 367-375, 1998.

2. Hu, J., Edsinger, A., Yi-Je, L., Donaldson, N., Solano, M., Solochek, A., Marchessault, R., "An advanced medical robotic system augmenting healthcare capabilities - robotic nursing assistant", IEEE International Conference on Robotics and Automation, pp. 6264-6269, 2011.

3. Cohen, J.E., "Human Population: The Next Half Century", Science, Vol. 302(5648), pp. 1172-1175, 2003.

4. Yu, H., Spenko, M., Dubowsky, S., "An Adaptive Shared Control System for an Intelligent Mobility Aid for the Elderly", Autonomous Robots, Vol. 15, pp. 53-66, 2003.

5. Morris, A., Donamukkala, R., Kapuria, A., Steinfeld, A., Matthews, J.T., Dunar-Jacob, J., Thrun, S., "A Robotic Walker that Provides Guidance", IEEE International Conference on Robotics and Automation, Vol. 1, pp. 25-30, Taipei, Taiwan, 14-19 September, 2003.

6. Balaguer, C., Gimenez, A., Jardon, A., Cabas, R., Correal, R., "Live Experimentation of the Service Robot Applications for Elderly People Care in Home Environments", IEEE International Conference on Robotics and Automation, pp. 2345 - 2350, Alberta, Canada, 2-6 August, 2005.

7. Arjohuntleigh Ceiling Lift, "Products:", http://www.arjohuntleigh.com/ukah/Products.asp?P ageNumber=3604\&ProductCategory_Id=15

8. Schaeffer, C., May, T., "Care-o-bot-a system for assisting elderly or disabled persons in home environments", Assistive Technology on the Threshold of the New Millennium, IOS Press, Amsterdam, 1999. 
9. Sato, T., Fukui, R., Morishita, H., Mori, T., "Construction of ceiling adsorbed mobile robots platform utilizing permanent magnet inductive traction method", Proceedings of IEEE/RSJ IROS, Vol. 1, pp. 552-558, 2004.

10. Wiener, J.M., Hanley, R.J., Clark, R., Van Nostra, J.F., "Measuring the activities of daily living: comparisons across national surveys", Journal of Gerontology, Social Sciences, Vol. 46, pp. 229-237, 1990.

11. Jain, R., Kasturi, R., Schunck, B.G., "Machine Vision", First Edition, McGraw-Hill, ISBN:0-07032018-7, New York,1995.

12. Georgoulas, C., Sirakoulis G.C., Andreadis, I., "Real-Time Stereo Vision Applications", Robot Vision, Ales Ude (Ed.), ISBN: 978-953-307-077-3, InTech, 2010.

13. Faugeras, O., "Three Dimensional Computer Vision: a geometric viewpoint", MIT Press, ASIN: B000OQHWZG, Cambridge, MA, 1993. 